\title{
Développement d'une approche couplée Automates Cellulaires - Eléments Finis pour la modélisation du développement des structures de grains en soudage TIG
}

\section{A coupled Cellular Automaton - Finite Element approach for the modelling of grain structure development in TIG welding}

\author{
Shijia Chen, Gildas Guillemot et Charles-André Gandin \\ CEMEF UMR CNRS 7635, Mines ParisTech, BP. 207, 06904 Sophia Antipolis, France
}

\begin{abstract}
In the welding area, the final properties of the weld bead are mainly induced by the grain structure developed during the melting and solidification steps. The mastery of the properties of the joining will be achieved with a better knowledge of the developed grain structure. A 3D coupled Cellular Automaton - Finite Element model is proposed in order to simulate the grains development in TIG process. This model is applied to the welding of a duplex stainless steel grade. The grain structure evolution is discussed for the various process parameters.
\end{abstract}

Résumé. Dans le domaine du soudage, les propriétés finales du cordon sont fortement liées à la structure de grains développée au cours des procédés de fusion / resolidification. La maîtrise des propriétés de l'assemblage final passe ainsi par une amélioration de la connaissance de sa structure de ce domaine. Dans cet objectif, un modèle couplé Automates Cellulaires - Eléments Finis est proposé pour simuler le développement, en volume, de cette structure, dans le cadre du soudage TIG. Ce modèle est appliqué au soudage d'acier Duplex 2202 et l'évolution de la structure de grains selon les paramètres procédés est discutée.

\section{INTRODUCTION}

La nécessité de réaliser des assemblages de grandes dimensions, liée aux contraintes de limiter les déformations finales, d'améliorer la durabilité des domaines soudés mais également de travailler sur des matériaux hétérogènes, a conduit à de profonds développement dans les procédés de soudage réalisés en milieux industriels. De nouvelles techniques ont ainsi été proposées (soudage par faisceau d'électrons, par laser, soudage hybride, ... ). De même, un intérêt s'est développé pour le soudage des alliages 'légers' (aluminium, magnésium ...) ou travaillants en milieux agressifs (acier inoxydables Duplex, alliage de Titane ...). Cependant, la détermination des propriétés d'usage du joint soudé passe par la connaissance de la métallurgie finale de ce cordon, et notamment de sa structure de grains. En effet, les critères de fissuration, les propriétés mécaniques locales, ou la détermination des défauts en CND sont liés à cette structure, tant en terme de taille de grains que d'orientation préférentielle de croissance. Dans ce cadre, un modèle couplé Automates Cellulaires Eléments Finis est proposé pour simuler le développement tridimensionnel des structures de grains, et l'apparition des orientations préférentielles de croissance. Ce modèle a été précédemment développé et appliqué à la solidification des alliages métalliques coulés [1-3]. Il est ici proposé de poursuivre ce développement en l'appliquant au soudage TIG d'un acier inoxydable Duplex.

\section{MODÈLE}

L'approche proposée, dénommée CAFE, est basée sur le couplage réalisé entre les deux approches Automates
Cellulaires (CA) - Elements-Finis (FE). Ainsi, à l'échelle du maillage éléments finis, l'équation de conservation de la chaleur est résolue, pour déterminer l'évolution macroscopique du champ d'enthalpie. Le chemin de solidification, liant température, fraction de solide et enthalpie nodales est calculé préalablement par le logiciel de calcul des équilibres thermodynamiques Thermo-Calc, en considérant un chemin de solidification de type Scheil [4]. A l'échelle de la grille de cellules cubiques, ce champ de températures est ainsi utilisé pour définir l'état des cellules et la cinétique de développement des formes de croissance qui leur est associées (Fig. 1).

Lors du processus de fusion, les cellules dont l'enthalpie dépasse celle associée au liquidus sont considérées liquides. Au cours du processus de resolidification, les cellules du métal de base, non fondues, servent de support à une croissance épitaxiale des grains du cordon. Ainsi, chaque grain est défini par l'ensemble de cellules qu'il recouvre, et dont le développement des formes de croissance modélise la croissance granulaire. Les alliages étudiés ayant une symétrie cubique (CC ou CFC), les directions de croissance préférentielles des pointes dendritiques sont les 6 directions $\langle 100\rangle$, représentants les 6 sommets de la forme de croissance. L'orientation spatiale de la forme de croissance associée à une cellule est définie par l'orientation cristallographique $\mathrm{du}$ grain auquel elle appartient, et repérée par les trois angles d'Euler $(\psi, \theta, \phi)$. Le recouvrement par la forme de croissance associée à une cellule $\mu$, du centre $C_{v}$ d'une cellule voisine $\nu$, libre de grains, permet de lui définir, par homothétie, une forme de croissance propre de même orientation spatiale. Les formes de croissance initiale, courante (Fig. 1) et maximale de la cellule, définissent 


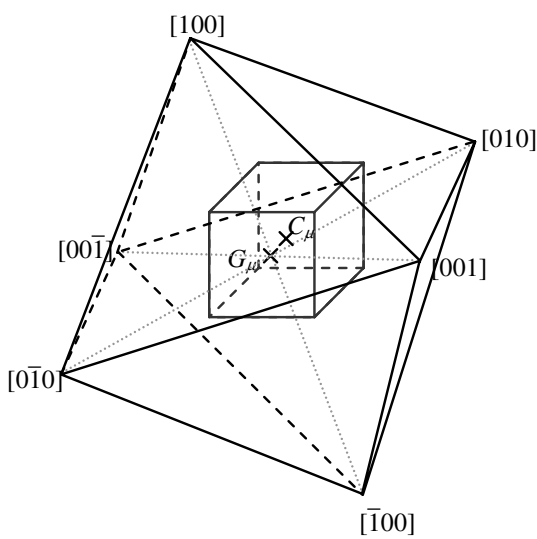

Figure 1. Cellule de la grille CA et forme de croissance associée. Le centre de la cellule et le centre de la forme de croissance sont respectivement les points $\mathrm{C}_{\mu}$ et $G_{u}$.

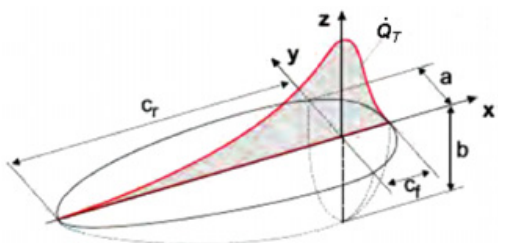

Figure 2. Source de chaleur volumique Gaussienne de type Goldak appliquée à la surface du système.

également la fraction de zone pâteuse cellulaire. Cette fraction est ensuite réassignée à l'échelle macro pour définir la fraction de zone pâteuse nodale. L'équation de conservation de la chaleur est ainsi résolue à cette échelle, avec la prise en considération de cette fraction pâteuse, réalisant le couplage entre approches $\mathrm{CA}$ et FE.

\section{RÉSULTAT ET DISCUSSION}

Le modèle développé a été appliqué à la simulation du soudage d'une tôle d'acier Duplex 2202 de forte épaisseur. Les dimensions des échantillons sont de $35 \times$ $15 \times 1,5 \mathrm{~cm}^{3}$. Le maillage FE destiné au calcul du champ de température est superposé au système. La grille de cellules CA est inclus dans le maillage $\mathrm{FE}$ et a une dimension de $24 \times 3 \times 1,2 \mathrm{~cm}^{3}$ (Fig. 2a). La taille des cellules est de $80 \mu \mathrm{m}$. Le maillage CA est destiné au calcul du développement de la structure de grains. Une structure initiale est donc simulée dans celui-ci, pour une densité de grains de $10^{11} \mathrm{~m}^{-3}$. La source de chaleur TIG est de type Goldak (Fig. 2). Aux frontières du domaine est appliquée une condition de refroidissement de type Fourier, avec un coefficient de transfert thermique de $300 \mathrm{~W} \cdot \mathrm{m}^{-1} \cdot \mathrm{K}^{-1}$, ainsi qu'un rayonnement thermique. La température extérieure est de $20^{\circ} \mathrm{C}$ sur l'ensemble des parois. La cinétique de croissance proposée pour les pointes de dendrite correspond est une loi parabolique, sous la forme $v=A \Delta T^{2}$, où $\Delta T$ est la surfusion locale. $A$ est pris égal à $10^{-7} \mathrm{~m} \cdot \mathrm{s}^{-1} \cdot \mathrm{K}^{-2}$.

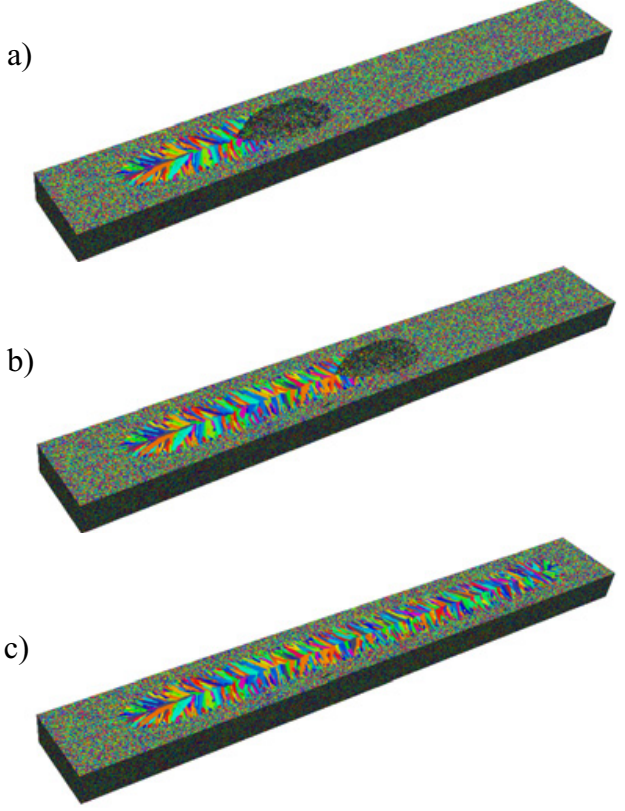

Figure 3. Structure de grains en soudage TIG, pour les temps de a) $40 \mathrm{~s}$, b) $60 \mathrm{~s}$ et c) $100 \mathrm{~s}$.

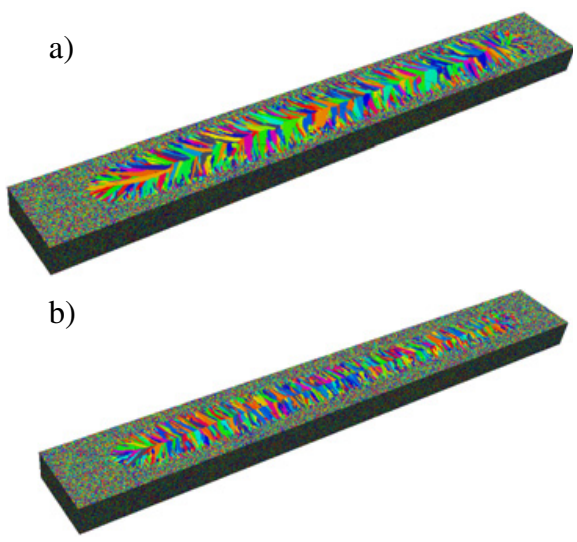

Figure 4. Structures de grains finale obtenues pour des puissances et vitesses de soudage de $4000 \mathrm{~W}$ et $1 \mathrm{~mm} \cdot \mathrm{s}^{-1}$, b) $15000 \mathrm{~W}$ et $5 \mathrm{~mm} \cdot \mathrm{s}^{-1}$.

La figure 3 présente l'évolution de la structure de grain pour une puissance TIG de $8000 \mathrm{~W}$, et une vitesse de déplacement de la source de $2 \mathrm{~mm} . \mathrm{s}^{-1}$, à différents temps de soudage. Ces figures montrent clairement le développement de la structure de grain, après refusion partielle de la structure initiale.

Les grains se développent transversalement, avec une faible inclinaison dans la direction longitudinale. Cette direction de croissance est guidée par le gradient de température locale, au cours de la solidification et correspond aux structures attendues [5]. La figure 4 présente les structures obtenues pour deux vitesses et deux sources de chaleur différentes.

On remarque l'évolution de la structure de grain selon la vitesse de déplacement de la source, pour une largeur de cordon comparable. Ainsi, aux faibles vitesses 
de déplacement, la structure de grains présente une orientation incurvée selon la direction longitudinale, en raison du gradient de température [5]. Aux plus fortes vitesses de déplacement, le bain liquide s'allonge, et le gradient de température est porté par la direction transversale. Ainsi, sur l'ensemble du cordon, on observe une structure fine de grains colonnaires.

\section{CONCLUSION}

Le modèle couplé Automates Cellulaires - Eléments Finis a été appliqué à la modélisation du développement des structures de grains en soudage TIG. Il montre précisément l'évolution de la structure de grains selon les paramètres procédés. Les évolutions de structures de grains sont conformes à celles attendues de la littérature. Elles doivent être confrontées par la suite aux résultats expérimentaux obtenus sur le même alliage Duplex 2202, pour valider l'approche proposée.

\section{Références}

[1] T. Carozzani, Ch.-A. Gandin, H. Digonnet, M. Bellet, K. Zaidat, Y. Fautrelle, Metall. Mater. Transactions A, 2013, Vol. 44A, 873-887

[2] T. Carozzani, H. Digonnet, Ch.-A. Gandin, Modell. Sim. Mater. Sc. Ing., 2012, Vol. 20, $\mathrm{N}^{\circ} 1$

[3] T. Carozzanni, Thèse de l'Ecole des Mines de Paris, Ecole doctorale SFA (2012)

[4] E. Scheil, Zeit. für Metallkunde, 1942, Vol. 34, 70-72

[5] D. A. Porter, K. E. Easterling, Phase Transformations in Metals and Alloys, $2^{\text {nd }}$ Ed., Chapman \& Hall, London, UK, 1992 